\title{
A DsbA-Deficient Periplasm Enables Functional Display of a Protein with Redox-Sensitive Folding on M13 Phage
}

Minyong Chen and James C. Samuelson

New England Biolabs, Inc., 240 County Road, Ipswich, Massachusetts 01938, United States

\section{Supporting Information}

\section{Methods and Materials.}

Materials and strains. Anti-HA antibody was purchased from Cell Signaling Technology (Beverly, MA). E. coli strains SHuffle T7 Express (C3029), T7 Express (C2566), NEB 5-alpha F'Iq (referred to as NEB5 $\alpha$ in the figures, C2992) and M13KO7 helper phage (N0315S) were from New England Biolabs (NEB). DHB4 and MB68 E. coli strains were from the NEB collection. Phagemid pComb3H was obtained from the Barbas laboratory at The Scripps Research Institute (La Jolla, CA).

DNA manipulation. All DNA constructs were made using the NEBuilder® HiFi DNA Assembly technique (NEB). To express SNAP-Fbs1 in E. coli cells, human Fbs1 gene encoding amino acid 93 to 296 was cloned into pSNAP-tag(T7) (NEB). This construct encodes SNAPFbs 1 fusion protein with SNAP tag at the N-terminus and Fbs1 (amino acid 93-296) at the Cterminus. The backbone of the Fbs1 phagemid construct is $\mathrm{pComb3H}$ where $\mathrm{pIII}$ fusion protein expression is controlled by the lac Z promoter/operator. The Fbs1 open reading frame (ORF) contains a heterologous signal sequence (ss), the human $F b s l$ gene encoding the sugar binding domain (amino acid 93 to 296), hemagglutinin (HA) epitope coding sequence, and gene III encoding the C-terminal domain (amino acid 230-406) of M13 pIII. The signal sequence of this fusion protein is cleaved by signal peptidase during membrane translocation, leaving mature Fbs1-HA-pIII (44 kDa) inserted in the inner membrane and anchored by pIII (Fbs1-HA is oriented into the periplasm).

Protein folding/solubility assay. Fbs1 tagged with SNAP (SNAP-Fbs1) was expressed in $E$. coli with an oxidizing cytoplasm (SHuffle T7 Express) or E. coli with a native reducing cytoplasm (T7 Express) at $37{ }^{\circ} \mathrm{C}$ or $30^{\circ} \mathrm{C}$ with $0.5 \mathrm{mM}$ IPTG induction. The cells were lysed in lysis buffer $(20 \mathrm{mM}$ Tris-HCl, pH7.5, $1 \mathrm{mM}$ EDTA, and $50 \mathrm{mM} \mathrm{NaCl})$ by sonication, and the total lysate was centrifuged at $16,000 \mathrm{x}$ g for $12 \mathrm{~min}$ at $4{ }^{\circ} \mathrm{C}$ to separate insoluble misfolded $\mathrm{Fbs} 1$ in pellet and soluble Fbs 1 in supernatant. Total lysate, pellet, and supernatant proteins were resolved by SDS-PAGE, and SNAP-Fbs1 protein was visualized by Coomassie blue staining.

Phage production and purification. Phage were produced according to Steiner et al. with some modifications $^{8}$. Briefly, Fbs1 phagemid constructs were transformed into indicated E. coli strains. E. coli cells harboring Fbs1 phagemids were grown in Luria-Broth (LB) medium supplemented with $1 \%$ glucose and $100 \mu \mathrm{g} / \mathrm{ml}$ ampicillin at $37^{\circ} \mathrm{C}$. When the culture reached $\mathrm{OD}_{600}=0.5 \sim 0.8$, the cells were supplemented with M13KO7 helper phage (E. coli cell: helper phage $=1: 20$ ) at $37^{\circ} \mathrm{C}$ for 30 min without agitation, and then with $250 \mathrm{rpm}$ agitation for $30 \mathrm{~min}$ 
at $37^{\circ} \mathrm{C}$. The culture medium was then replaced by LB medium supplemented with $100 \mu \mathrm{g} / \mathrm{ml}$ ampicillin, $40 \mu \mathrm{g} / \mathrm{ml}$ kanamycin, and $100 \mu \mathrm{M}$ isopropyl- $\beta$-D-thiogalactoside (IPTG). The cells were then grown at $25^{\circ} \mathrm{C}$ for $16-18 \mathrm{hrs}$ with $250 \mathrm{rpm}$ agitation. The culture medium containing phage were collected, and incubated on ice for more than $1 \mathrm{hr}$ with one-fourth volume of $20 \%$ PEG8000/ $2.5 \mathrm{M} \mathrm{NaCl}$ to precipitate phage. The phage were collected by centrifugation at 5,600 $\mathrm{x}$ g for $20 \mathrm{~min}$ at $4{ }^{\circ} \mathrm{C}$. The collected phage were re-dissolved in $1 \mathrm{ml}$ of buffer A $(20 \mathrm{mM}$ Tris$\mathrm{HCl}, \mathrm{pH} 7.5,50 \mathrm{mM} \mathrm{NaCl}, 1 \mathrm{mM}$ EDTA), and precipitation with PEG8000/ $2.5 \mathrm{M} \mathrm{NaCl}$ was repeated twice to further purify the phage. The titer of M13 phage was determined using the equation: $\left(\mathrm{A}_{269}-\mathrm{A}_{320}\right) \times 6 \times 10^{16} /$ number of bases per phagemid = phage number $/ \mathrm{ml} . \mathrm{A}_{269}$ and $\mathrm{A}_{320}$ of the phage were measured by a NanoDrop Spectrophotometer (Thermo Fisher Scientific, Waltham, MA). Anti-HA immunoblotting was used to determine whether Fbs1 can be displayed on the phage. The phage were solubilized by SDS sample buffer and phage proteins were resolved by SDS-PAGE, followed by anti-HA immunoblotting.

Preparation of RNase B beads. Glycoprotein RNase B (NEB) was conjugated to Affi-Gel 10 beads (BioRad, Hercules, CA) ${ }^{14,15}$. After conjugation, the RNase B beads were incubated with 6 $\mathrm{M}$ guanidine hydrochloride to denature RNase B. After washing with 30x bead volume of water to remove guanidine hydrochloride, the RNase B beads were used to pull down the phage displaying functional/properly folded Fbs1.

RNase B pull-down assay. Equal amounts (approx. $5 \times 10^{11} \mathrm{cfu}$ ) of phage displaying Fbs1 were incubated with RNase B beads or control beads (non-conjugated Affi-Gel 10 beads with conjugation groups blocked) in binding buffer ( $20 \mathrm{mM}$ Tris-HCl, pH7.5, $1 \mathrm{mM}$ EDTA, $50 \mathrm{mM}$ $\mathrm{NaCl}, 20 \%$ Pierce Protein-Free Blocking Buffer (Thermo Fisher Scientific)) at $4{ }^{\circ} \mathrm{C}$ for $1 \mathrm{hr}$. After incubation, the beads were washed twice with wash buffer $(20 \mathrm{mM}$ Tris- $\mathrm{HCl}, \mathrm{pH} 7.5,1 \mathrm{mM}$ EDTA, $50 \mathrm{mM} \mathrm{NaCl}$ ), and then incubated with SDS sample buffer and heated to elute bound phage. The eluents were subjected to anti-HA immunoblot to detect the bound phage displaying functional Fbs1. 


\section{Supplemental Figures}

A

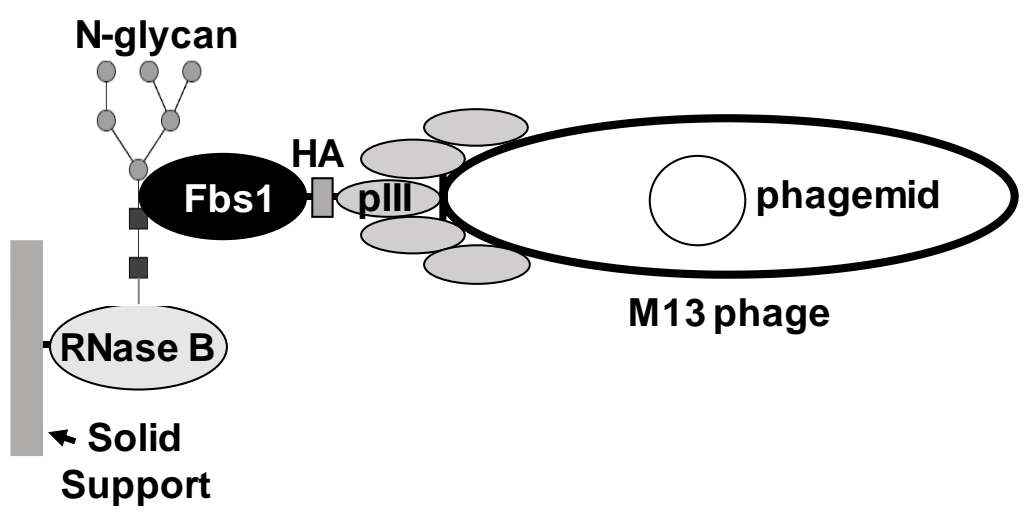

B

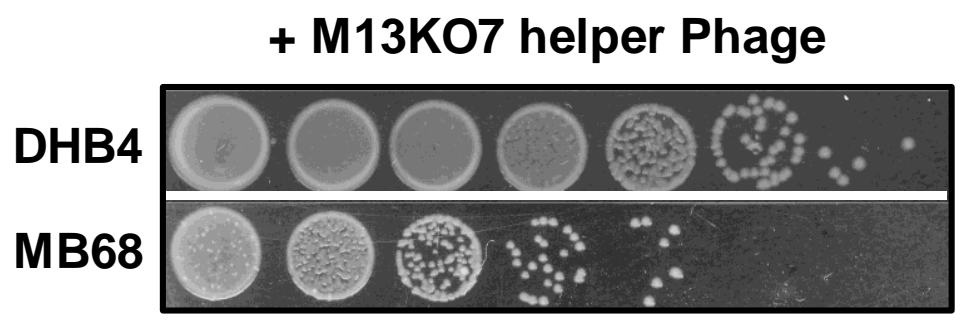

Figure S1

A. Illustration of the pull-down assay to detect M13 phage displaying properly folded/functional Fbs1. Fbs1 tagged with the HA epitope at its C-terminus is fused to C-terminus of M13 pIII. The fusion protein of Fbs1-HA-pIII is incorporated into M13 phage. Fbs1 is a carbohydrate binding protein, and it recognizes high mannose $\mathrm{N}$-glycans at a sub-micromolar $\mathrm{K}_{\mathrm{d}}$. RNase $\mathrm{B}$, a glycoprotein with a high mannose $\mathrm{N}$-glycan, is immobilized in a solid support such as Affi-Gel 10 beads. Only properly folded/ functional Fbs1 recognizes and binds to the N-glycan of RNase B and therefore will be pulled down by immobilized RNase B (RNase B beads). Anti-HA immunoblotting is then used to detect the bound M13 phage.

B. MB68 shows lower infection efficiency by M13 helper phage. MB68 and its isogenic $d s b A+$ parental strain DHB4 were infected with M13KO7 helper phage (harboring a kanamycin resistance gene). After infection, $E$. coli cells were subjected to 10-fold serial dilutions (from left to right), and spotted on LB agar plates with $40 \mu \mathrm{g} / \mathrm{ml}$ kanamycin. The plate was incubated at 37 ${ }^{\circ} \mathrm{C}$ overnight and then photographed. 